\title{
Demografía del caracol Plicopurpura pansa (Neotaenioglossa: Muricidae) y constitución de la comunidad malacológica asociada en Guerrero, México
}

\author{
Rafael Flores-Garza ${ }^{1}$, Pedro Flores-Rodríguez ${ }^{1}$, Sergio García-Ibáñez ${ }^{1}$ \\ \& Arcadio Valdés-González ${ }^{2}$ \\ 1 Unidad Académica de Ecología Marina, Universidad Autónoma de Guerrero, Acapulco, Guerrero, México. Gran Vía \\ Tropical \#20 Fraccionamiento las Playas, Acapulco, Guerrero, México. C.P. 39390. Tel.: 744 4832780; \\ rfloresgarza@yahoo.com \\ 2 Facultad de Ciencias Biológicas, Universidad Autónoma de Nuevo León, San Nicolás de los Garza, Nuevo León, \\ México. Ciudad Universitaria. C.P. 66450. San Nicolás de los Garza, Nuevo León, México. Tel.: 818 3520779; \\ acuacu_uanl@yahoo.com.mx
}

Recibido 11-I-2006. Corregido 09-V-2006. Aceptado 15-I-2007.

\begin{abstract}
Demography of the snail Plicopurpura pansa (Neotaenioglossa: Muricidae) and constitution of the associated mollusk community, in Guerrero, Mexico. We quarterly sampled the molluskan community of Playa Ventura, Guerrero, Mexico. Sample size $\left(20 \mathrm{~m}^{2}\right.$ in $1 \mathrm{~m}^{2}$ units $)$ was determined by the spatial distribution pattern of Plicopurpura pansa using a negative binomial distribution. The density of the P. pansa was 5.19 snails $/ \mathrm{m}^{2}$ and the sex ratio near 1:1. The average size was $20.10 \mathrm{~mm}$ (most females were 10.5 to $27.0 \mathrm{~mm}$; most males 12.0 to $27.0 \mathrm{~mm}$ ). Density was high and small sized dominated. Mean size varied with time. General mean weight was $1.79 \mathrm{~g}$. Females weight varied more, maybe because of the reproductive cycle. Recruits were collected in five samplings. Most copulation activity was in March of 2001. The associated community includes 26 species of Gastropoda, five of Bivalvia and one of Polyplacophora, Brachidontes semilaevis being dominant. Rev. Biol. Trop. 55 (3-4): 867-878. Epub 2007 December, 28.
\end{abstract}

Key words: Plicopurpura pansa, population, density, sizes, recruitment, mollusks community.

En la costa del Pacífico Mexicano, existen extensiones importantes de zonas rocosas donde habita el caracol Plicopurpura pansa (Gould, 1853). Su distribución abarca de Bahía Magdalena, Baja California Sur hasta Colombia e Islas Galápagos (Keen 1971) y de acuerdo con los criterios de zonación de Stephenson y Stephenson (1949), la franja del litoral donde vive es la mesolitoral (Villalpando 1986, León 1989).

El caracol púrpura es un recurso cultural y económicamente importante, debido a que de el se extrae un tinte que desde épocas prehispánicas se ha utilizado para teñir prendas de vestir, las cuales han estado relacionadas con el poder y la cultura mágico-religiosa de los grupos indígenas que se han dedicado a esta actividad (Turok et al. 1988).
En la primera mitad de la década de los ochentas, una compañía japonesa contrató personas ajenas a la actividad de tinción para recoleccionar la tinta o teñir los hilos, producto que era enviado al Japón para elaborar kimonos o tapicería. Debido al mal manejo y a las técnicas inapropiadas de ordeña, la población se redujo y los teñidores indígenas fueron desplazados (Turok y Acevedo 1999). En 1983, un grupo de teñidores de la región de Pinotepa de Don Luis Oaxaca, denunciaron el manejo inadecuado del recurso y los daños a la población de este molusco, lo que llevó a que en 1988 apareciera en el Diario Oficial de la Federación un acuerdo para regular la conservación y aprovechamiento del recurso púrpura (Anónimo 1988). Actualmente se le considera como una especie sujeta a protección especial 
con base en la norma oficial mexicana NOM059-ecol-1994 (Anónimo 1994).

A partir de esta problemática, se han desarrollado estudios sobre este caracol que se han enfocado al análisis de la estructura de la población, aspectos reproductivos y fauna asociada al caracol púrpura. Entre estos se encuentran los realizados por Villalpando (1986), Hernández y Acevedo (1987), Acevedo et al. (1987), Salcedo et al. (1988), Álvarez (1989), León (1989), Acevedo et al. (1990), Holguín (1993), Castillo-Rodríguez y Amezcua-Linares (1992), Ramos-Cruz (1993), Reyes (1993), Acevedo y Escalante (1993), García (1994), Flores (1995), Enciso et al. (1998), Michel (2000), Michel-Morfin et al. (2000), MichelMorfin et al. (2002) y Ramírez-Rodríguez y Naegel (2003).

El objetivo fue analizar la estructura de la población del $P$. pansa, con base en la densidad, proporción por sexos, tallas y pesos y conocer la malacofauna con la que principalmente se asocia el caracol púrpura.

\section{MATERIALES Y MÉTODOS}

Metodología de campo: El estudio se realizó en el mesolitoral rocoso de Playa Ventura $\left(16^{\circ} 32^{\prime} 8.3^{\prime \prime} \mathrm{N}\right.$ y $98^{\circ} 54^{\prime} 47.6^{\prime \prime} \mathrm{W}$ a $16^{\circ} 32^{\prime} 3.95^{\prime \prime}$ $\mathrm{N}$ y $\left.98^{\circ} 54^{\prime} 21.53^{\prime \prime} \mathrm{W}\right)$, entre septiembre 2000 a diciembre 2001, con recolectas trimestrales. El área de muestreo fue de $20 \mathrm{~m}^{2}$ y se determinó a partir del grado de dispersión espacial (Krebs 1999) del P. pansa, con nivel de confianza de $90 \%$ y error estándar de $30 \%$. Para las recolectas, se empleó un transecto de $30 \mathrm{~m}$ de largo con $2 \mathrm{~m}$ de amplitud, el cual se colocó paralelo a la línea de costa. La unidad de muestreo fue un cuadrante de $1 \mathrm{~m}$ de lado. El muestreo fue sistemático (Scheaffer et al. 1987), seleccionando aleatoriamente el punto de partida y colocando el cuadrante en la parte inferior del transecto. Terminada la primera unidad de muestreo, el cuadrante se colocó inmediatamente encima. Al concluir ambas unidades de muestreo se dejó un espacio de dos metros y se establecieron los nuevos cuadrantes.
Los P. pansa que quedaron dentro de la unidad de muestreo, fueron recoleccionados, separados por sexo, contados y medidos en largo $(\mathrm{mm})$ desde la punta del ápice hasta la abertura del canal sifonal, además se pesaron (g) y se registró el número de parejas que se encontraron copulando. Al terminar la toma de datos los caracoles fueron devueltos a su hábitat. Los moluscos encontrados dentro de los cuadrantes también fueron recoleccionados (solo los necesarios), identificados y cuantificados. La adecuada ubicación taxonómica requirió de una fase de confirmación en el laboratorio, mediante el uso de claves especializadas como, Morris (1966), Keen (1971) y Reyes-Gómez y Salcedo-Vargas (2002), y se actualizó la nomenclatura de acuerdo con Skoglund (1991, 1992). El análisis de la fauna se basó en la información de las mismas recolecciones con excepción de diciembre de 2000 .

Análisis de datos: Se calculó para el $P$. pansa la densidad en caracoles $/ \mathrm{m}^{2}$, la proporción sexual (hembra:macho), el promedio y coeficiente de variación de talla y peso (Steel y Torrie 1997). Éstas estimaciones se realizaron para el total de organismos, así como para hembras y machos de manera independiente, calculándose para las seis fechas en su conjunto y por cada fecha de recolecta.

La comparación de la densidad de caracoles entre las fechas de recolecta, se llevó a cabo mediante un Análisis de Varianza de una sola vía (ANOVA) $(\mathrm{p} \leq 0.05)$ (Daniel 2002) y la comparación múltiple de medias se realizó con la prueba $\mathrm{C}$ de Dunnett.

Para comprobar la igualdad de proporciones entre sexos se aplicó la prueba de homogeneidad de chi cuadrada ( $\mathrm{p} \leq 0.05$ ) (Daniel 2002). Las tallas mejor representadas y el análisis de reclutamiento de la población de $P$. pansa se obtuvieron a partir de histogramas de distribución de frecuencias.

Para el conjunto de datos de talla en hembras y en machos, se buscaron diferencias entre los promedios por fecha de recolección, mediante un análisis de varianza de una vía ( $p$ $\leq 0.05$ ). La homogeneidad de las varianzas se 
comprobó con la prueba de Levene $(\mathrm{p} \leq 0.05)$. Los grupos por tallas promedio se formaron con base en la comparación múltiple de medias $\mathrm{C}$ de Dunnett.

Para la comunidad malacológica, los datos se analizaron de la siguiente manera: La riqueza se consideró como el número de especies de moluscos presentes. El análisis de Dominancia Simple se basó en el total de organismos de cada especie con respecto al total de organismos de todas las especies, expresada en porcentaje. La densidad se evaluó en organismos $/ \mathrm{m}^{2}$. La determinación de las especies mayormente asociadas al $P$. pansa se realizó tomando como criterio a aquellas que presentaron densidad de al menos 1.0 organismo $/ \mathrm{m}^{2}$.

\section{RESULTADOS}

Se recoleccionaron en total 623 caracoles púrpura (316 hembras y 307 machos). La densidad para todo el ciclo fue $5.19 \pm 4.63$ organismos $/ \mathrm{m}^{2}$ y varió de $3.85 \pm 3.24$ organismos $/ \mathrm{m}^{2}$ a $8.55 \pm 6.97$ organismos $/ \mathrm{m}^{2}$. El análisis de las densidades por fecha evidenció diferencias significativas (ANOVA $\mathrm{p}<0.05$ ) y se formaron dos grupos. El primero lo constituyeron las densidades de las recolectas de septiembre y diciembre 2000 así como marzo, junio y septiembre de 2001; mientras que el segundo grupo que correspondió a las densidades mayores, estuvo integrado por las recolectas de junio $\mathrm{y}$ diciembre 2001.

La proporción hembramacho fue de 1:0.80, oscilando por fecha de 0.70:1 a 1:0.80, en ningún caso se registraron diferencias significativas (chi cuadrada $\mathrm{p}>$ 0.05) (Cuadro 1).

La talla promedio estimada para todos los organismos fue de $20.10 \pm 6.01$ $\mathrm{mm}$, mientras que en hembras de $19.68 \pm 6.83 \mathrm{~mm}$ y en machos de $20.53 \pm 5.01 \mathrm{~mm}$.
El coeficiente de variación en talla en hembras fue de $34.71 \%$, mientras que en machos fue de $24.40 \%$. La talla más pequeña fue $7.30 \mathrm{~mm}$ y se registró en machos y la máxima fue de 40.80 mm y se presentó en hembras (Cuadro 2). Para hembras y machos, se encontraron diferencias significativas entre las tallas promedio de cada una de las fechas (Fisher $\mathrm{p}<0.05$ ), formándose dos grupos de medias para cada sexo (Cuadro 3). En ambos sexos los datos correspondientes a diciembre de 2000 y 2001 se encuentran en el grupo con los menores promedios y junio en el grupo de mayor promedio. Las tallas mejor representadas estuvieron entre los 10.5 a $27.0 \mathrm{~mm}$ en hembras y de 12.5 a $27.0 \mathrm{~mm}$ en machos.

El peso promedio para todos los organismos de $P$. pansa fue de $1.79 \pm 1.68 \mathrm{~g}$, las hembras presentaron un peso promedio de $1.78 \pm$ $1.94 \mathrm{~g}$ y los machos de $1.81 \pm 1.36 \mathrm{~g}$. Los datos de peso del caracol púrpura presentaron mayor variabilidad con respecto a los de longitud, observándose en hembras un coeficiente de variación de $108.9 \%$ y en machos de $75.13 \%$. En las hembras, en la mayoría de las fechas de recolección, el coeficiente de variación en peso fue igual o mayor que el $100 \%$, solo en junio se presentó una disminución importante de este coeficiente, siendo de $50.59 \%$ (Cuadro 2, Fig. 1).

Se observó reclutamiento en cinco fechas de recolección. En septiembre y diciembre 2000 y 2001 se presentó la mayor frecuencia

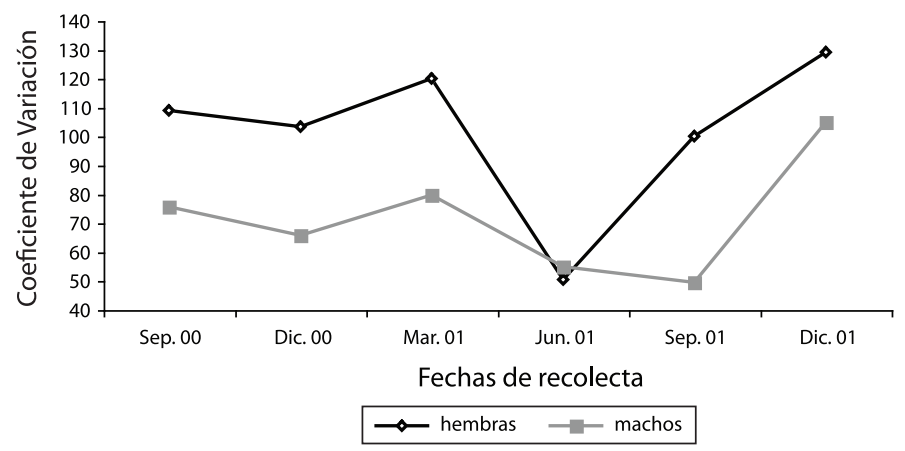

Fig. 1. Coeficiente de Variación en peso de Plicopurpura pansa, por fecha, en Playa Ventura, Guerrero, México.

Fig. 1. Variation coefficient for weight, Plicopurpura pansa, by dates, at Playa Ventura, Guerrero, México. 
CUADRO 1

Densidad y proporción sexual del caracol Plicopurpura pansa, por fechas de recolección en Playa Ventura, Guerrero, México (septiembre 2000 a diciembre 2001)

TABLE 1

Density and sex ratio of the snail Plicopurpura pansa, by sampling date, Playa Ventura, Guerrero, Mexico (September 2000 to December 2001)

\begin{tabular}{|c|c|c|c|c|}
\hline Fecha & Sexo & $\mathrm{n}$ & $\begin{array}{l}\text { Densidad } \\
\text { org./ } \mathrm{m}^{2}\end{array}$ & $\begin{array}{l}\text { Proporción sexual } \\
\text { (H:M) }\end{array}$ \\
\hline \multirow[t]{3}{*}{ Todas } & Ambos & 623 & $5.19 \pm 4.63$ & $1: 0.97$ \\
\hline & Hembras & 316 & $2.63 \pm 2.75$ & \\
\hline & Machos & 307 & $2.56 \pm 2.50$ & \\
\hline \multirow[t]{3}{*}{ Sep. 00} & Ambos & 77 & $3.85 \pm 3.24$ & $1: 0.92$ \\
\hline & Hembras & 40 & $2.00 \pm 1.78$ & \\
\hline & Machos & 37 & $1.85 \pm 2.21$ & \\
\hline \multirow[t]{3}{*}{ Dic. 00} & Ambos & 81 & $4.05 \pm 4.31$ & $0.97: 1$ \\
\hline & Hembras & 40 & $2.00 \pm 2.03$ & \\
\hline & Machos & 41 & $2.05 \pm 2.59$ & \\
\hline \multirow[t]{3}{*}{ Mar. 01} & Ambos & 87 & $4.35 \pm 3.67$ & $0.70: 1$ \\
\hline & Hembras & 36 & $1.80 \pm 1.67$ & \\
\hline & Machos & 51 & $2.55 \pm 2.69$ & \\
\hline \multirow[t]{3}{*}{ Jun. 01} & Ambos & 109 & $5.45 \pm 3.99$ & $1: 0.91$ \\
\hline & Hembras & 57 & $2.85 \pm 2.66$ & \\
\hline & Machos & 52 & $2.60 \pm 2.14$ & \\
\hline \multirow[t]{3}{*}{ Sep. 01} & Ambos & 98 & $4.90 \pm 3.37$ & $0.96: 1$ \\
\hline & Hembras & 48 & $2.40 \pm 2.30$ & \\
\hline & Machos & 50 & $2.50 \pm 1.91$ & \\
\hline \multirow[t]{3}{*}{ Dic. 01} & Ambos & 171 & $8.55 \pm 6.97$ & $1: 0.80$ \\
\hline & Hembras & 95 & $4.75 \pm 4.32$ & \\
\hline & Machos & 76 & $3.80 \pm 3.11$ & \\
\hline
\end{tabular}

En ningún caso hubo diferencia significativa entre los sexos. 
CUADRO 2

Tallas y pesos del caracol Plicopurpura pansa por fechas de recolección en Playa Ventura, Guerrero, México (septiembre 2000 a diciembre 2001)

TABLE 2

Sizes and weights of the snail Plicopurpura pansa per sampling date, Playa Ventura, Guerrero, Mexico (September 2000 to December 2001)

Longitud (mm)

Fecha Sexo $n$ Media Mín. Máx C. V.

Todas

ก 307

Tot 623

$20.53 \pm 5.01$

7.30

33.80

24.40

$20.10 \pm 6.01$

7.30

40.80

29.90

ㅇ 40

Sep. 00

$$
\text { 万ิ } 37
$$

$20.22 \pm 8.19$

9.30

36.00

40.50

Tot 7

Dic. 00

$$
\text { o }
$$

Tot

$$
21.05 \pm 5.28
$$

12.80

33.40

$\begin{array}{lll}9.30 & 36.00 \quad 33.57\end{array}$

40

$17.35 \pm 6.22$

8.40

34.20

35.85

$\begin{array}{lll}12.10 & 27.60 & 23.70\end{array}$

$19.53 \pm 4.63$

$8.40 \quad 34.20 \quad 30.06$

․ 36

$21.80 \pm 7.54$

11.2

40.40

34.58

Mar. 01

万' 51

$20.77 \pm 5.37$

7.30

33.10

25.85

To

87

$21.20 \pm 6.34 \quad 7.30$

$40.40 \quad 29.90$

57

$22.96 \pm 3.59$

$13.70 \quad 36.50$

15.71

Jun. 01

万人 52

Tot

$22.74 \pm 3.61$

15.3

32.50

15.87

$13.70 \quad 36.50 \quad 15.66$

ㅇ 48

Sep. 01

Tot

Tot 98

$$
20.86 \pm 7.47
$$

$40.80 \quad 35.81$

$20.88 \pm 4.60$

$$
8.50
$$

29.70

22.03

$8.50 \quad 40.80 \quad 29.46$

우 $\quad 95 \quad 17.07 \pm 6.12$

$9.50 \quad 36.30 \quad 35.85$

$0^{x} \quad 76$

Tot 171

$18.91 \pm 5.36$

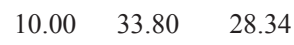

$17.89 \pm 5.85$

$9.50 \quad 36.30 \quad 32.69$
Peso (g)

Media Mín. Máx

C. V.

08.9

$\begin{array}{llll}1.81 \pm 1.36 & 0.10 & 9.70 & 75.13\end{array}$

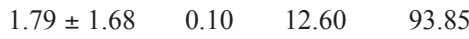

$\begin{array}{llll}2.01 \pm 2.19 & 0.10 & 7.80 & 108.9\end{array}$

$\begin{array}{llll}1.98 & \pm 1.50 \quad 0.40 \quad 7.10 & 75.75\end{array}$

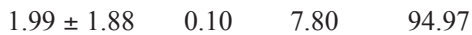

$\begin{array}{llll}1.22 \pm 1.59 & 0.10 & 6.60 & 103.3\end{array}$

$\begin{array}{llll}1.46 \pm 0.96 & 0.30 & 3.70 & 65.75\end{array}$

$\begin{array}{llll}1.34 & \pm 1.31 \quad 0.10 & 6.60 & 97.76\end{array}$

$2.45 \pm 2.94 \quad 0.30 \quad 12.60$

120.0

$\begin{array}{llll}1.88 & \pm 1.50 \quad 0.10 & 6.60 & 79.78\end{array}$

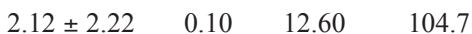

$2.17 \pm 1.10 \quad 0.50 \quad 7.40 \quad 50.59$

$2.17 \pm 1.19 \quad 0.60 \quad 6.60 \quad 54.83$

$2.17 \pm 1.14 \quad 0.50 \quad 7.40 \quad 52.53$

$2.09 \pm 2.09 \quad 0.10 \quad 11.70 \quad 100.0$

$1.86 \pm 0.92 \quad 0.10 \quad 4.50 \quad 49.46$

$1.97 \pm 1.60 \quad 0.10 \quad 11.70 \quad 81.21$

$1.27 \pm 1.64 \quad 0.10 \quad 11.00$

129.1

$1.58 \pm 1.66 \quad 0.20 \quad 9.70 \quad 105.0$

$1.40 \pm 1.65 \quad 0.10 \quad 11.00 \quad 117.8$ 
CUADRO 3

Grupos de tallas promedio por fecha de recolecta en hembras y machos del caracol Plicopurpura pansa en Playa Ventura, Guerrero, México

TABLE 3

Average sizes groups per sampling date, for females and males of the snail Plicopurpura pansa, Playa Ventura, Guerrero, Mexico

\begin{tabular}{|c|c|c|c|}
\hline \multirow[t]{2}{*}{ Sexo } & \multirow[t]{2}{*}{ Fecha } & \multicolumn{2}{|c|}{$\begin{array}{l}\text { Comparación múltiple } \\
\text { de medias: } \\
\text { Grupos C de Dunnett }\end{array}$} \\
\hline & & 1 & 2 \\
\hline \multirow{6}{*}{ q } & Dic. 01 & 17.07 & \\
\hline & Dic. 00 & 17.35 & \\
\hline & Sep. 00 & & 20.22 \\
\hline & Sep. 01 & & 20.86 \\
\hline & Mar. 01 & & 21.80 \\
\hline & Jun. 01 & & 22.96 \\
\hline \multirow{6}{*}{$0^{\pi}$} & Dic. 01 & 18.91 & \\
\hline & Dic. 00 & 19.53 & \\
\hline & Mar. 01 & 20.77 & 20.77 \\
\hline & Sep. 01 & 20.88 & 20.88 \\
\hline & Sep. 00 & 21.05 & 21.05 \\
\hline & Jun. 01 & & 22.74 \\
\hline
\end{tabular}

de reclutas. En marzo disminuyó la frecuencia de organismos en tallas pequeñas y en junio no se encontraron organismos con tallas inferiores a los $13.5 \mathrm{~mm}$ (Fig. 2). El mayor número de parejas copulando se registró en la recolecta de marzo, mientras que en otras fechas de manera ocasional se presentó alguna pareja copulando.

En Playa Ventura se encontraron 34 especies de moluscos, pertenecientes a 23 géneros y 16 familias. La riqueza de especies se distribuyó de la siguiente manera: en diciembre de 2000 se registraron 18 especies. En marzo, junio, septiembre y diciembre de 2001 se encontraron $25,27,23$ у 22 especies, respectivamente. Se identificaron 26 especies de gasterópodos, siete de bivalvos y una de poliplacóforos. Las especies con mayor dominancia simple fueron Brachidontes semilaevis (Menke, 1849) y Choromytilus palliopunctatus (Carpenter, 1857).

En el ciclo anual 14 especies presentaron densidad igual o mayor a 1.0 organismo $/ \mathrm{m}^{2}$, las especies consideradas como las mayormente asociadas al caracol púrpura fueron: $B$. semilaevis, $C$. palliopunctatus, Nodilittorina aspera (Philippi, 1846), Nerita scabricosta Lamark, 1822, Isognomon janus Carpenter, 1857, Nodilittorina modesta (Philippi, 1846), Petaloconchus complicatus Dall, 1908, Chama echinata Broderip, 1835, Hoffmannola hansi Marcus \& Marcus, 1967, Lottia mitella (Menke, 1847), Petaloconchus macrophragma Carpenter, 1857, Chiton articulatus Sowerby, 1832, Fissurella gemmata Menke, 1847 y Mancinella triangularis (Blainville, 1832)(Cuadro 4).

\section{DISCUSIÓN}

La densidad de $P$. pansa registrada en Playa Ventura, es mayor a lo que se reporta para otros Estados del Pacífico Mexicano. La mayor densidad ha sido reportada por Flores (1995) en Acapulco, Guerrero. Acevedo et al. (1996), mencionaron que en la costa del Pacífico Mexicano la densidad se incrementa conforme al aumento en la latitud, y refieren que los tres Estados que están hacia el norte (Jalisco, Nayarit y Sinaloa) son los que presentan mejores condiciones en cuanto a densidad. Según los resultados de densidad reportados por Flores (op cit.) y lo encontrado en el presente estudio, permite concluir que no es posible definir un patrón de densidad para este molusco con base en la latitud, ya que dos sitios del Estado de Guerrero muestreados en diferentes años, presentaron densidades mayores a lo reportado en Estados que se encuentran en latitudes superiores.

En Playa Ventura, la proporción hembra-macho en la población del caracol púrpura, presentó tendencia a la unidad (1.0:1.0), al respecto Michel (2000), en diferentes playas determinó un comportamiento similar en la 

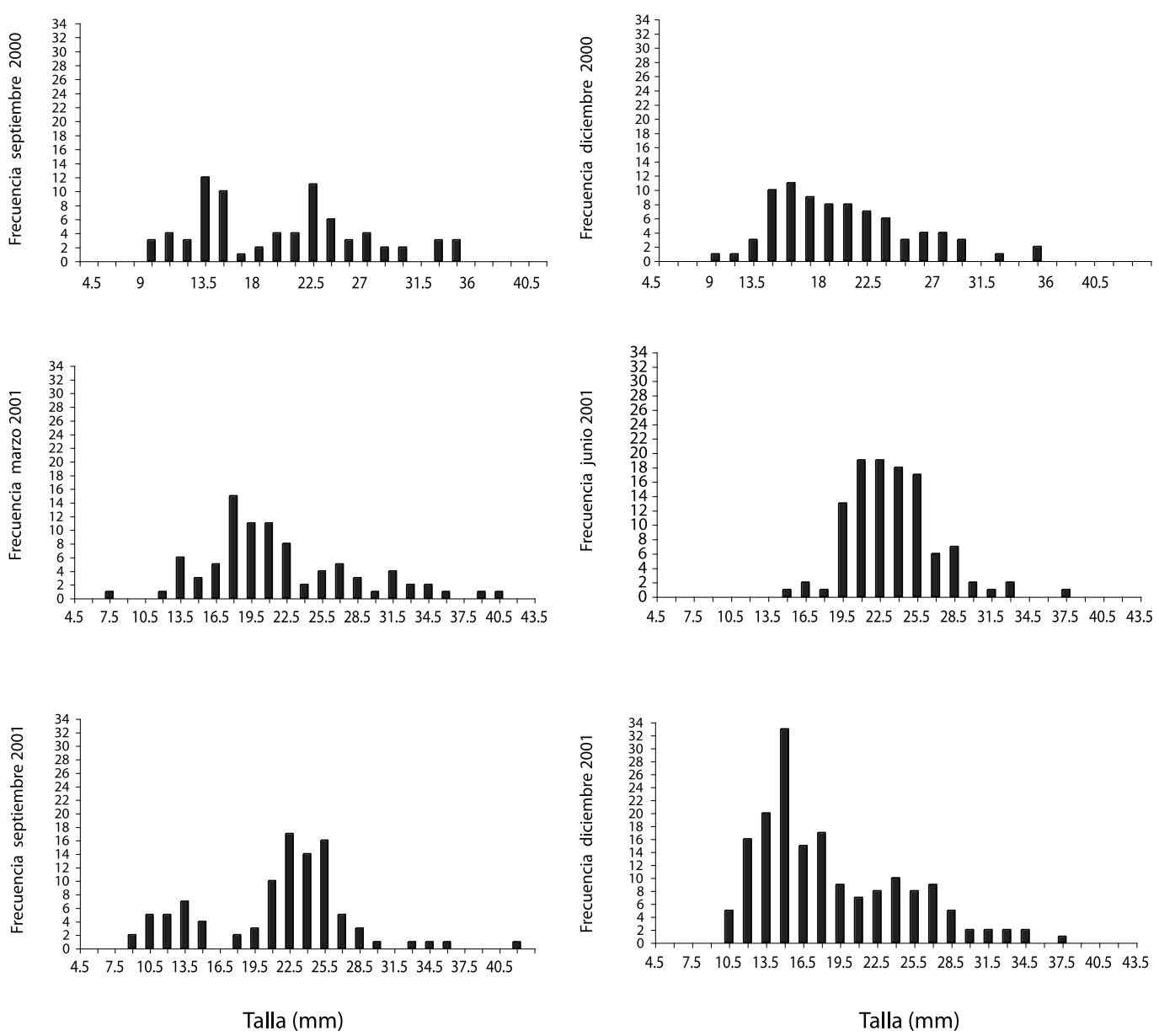

Fig. 2. Tallas según fecha de recolección de Plicopurpura pansa en Playa Ventura, Guerrero.

Fig. 2. Size per date for Plicopurpura pansa in Playa Ventura, Guerrero.

proporción por sexos; sin embargo, Acevedo et al. (1996), menciona que los machos a lo largo de las costas del Pacífico Mexicano, son en todos los casos más abundantes que las hembras. Holguín (1993), también indicó una mayor cantidad de machos, donde estos duplican a las hembras. Por otra parte, Michel (op cit.) determinó para Baja California Sur un macho por cada 1.85 hembras, a pesar de esto, en muchos sitios donde estudiaron la proporción sexual han reportado proporciones que se aproximan a 1.0:1.0.

Las tallas promedio del caracol púrpura encontradas en Playa Ventura, fueron menores a las reportadas en otros lugares del Pacífico Mexicano. La talla promedio en hembras y machos solo fue superior a la que reportó Flores (1995). Las hembras registraron las mayores tallas individuales, lo que ha sido reportado por todos los trabajos que analizan este aspecto. Durante el ciclo de estudio se observaron variaciones en las tallas promedio para hembras y machos, y se percibió que en las recolectas de diciembre de 2000 y 2001 se ubican las menores tallas promedio.

La mayoría de los P. pansa en Playa Ventura, se encontraron congregados en un intervalo de tallas que va de los 10.5 a los $27.0 \mathrm{~mm}$. En 
CUADRO 4

Especies de moluscos, densidad y dominancia en el mesolitoral rocoso de Playa Ventura, Guerrero

TABLE 4

Species of mollusks, density and dominancy in the middle intertidal zone at Playa Ventura, Guerrero

\section{Especies}

Brachidontes semilaevis (Menke, 1849)

Choromytilus palliopunctatus (Carpenter, 1857)

Nodilittorina aspera (Philippi, 1846)

Nerita scabricosta Lamark, 1822

Isognomom janus Carpenter, 1857

Nodilittorina modesta (Philippi, 1846)

Plicopurpura pansa (Gould, 1853)

Petaloconchus complicatus Dall, 1908

Chama echinata Broderip, 1835

Hoffmannola hansi Marcus \& Marcus, 1967

Lottia mitella (Menke, 1847)

Petaloconchus macrophragma Carpenter, 1857

Chiton articulatus Sowerby, 1832

Fissurella gemmata Menke, 1847

Mancinella triangularis (Blainville, 1832)

Siphonaria palmata Carpenter, 1857

Fissurella nigrocincta Carpenter, 1856

Thais kiosquiformis (Duclos, 1832)

Lottia acutapec (Berry, 1960)

Siphonaria gigas Sowerby, 1825

Striostrea prismatica (Gray, 1825)

Columbella fuscata Sowerby, 1832

Lottia mesoleuca (Menke, 1851)

Fissurella asperella Sowerby, 1835

Saccostrea palmula (Carpenter, 1857)

Eulithidium phasianella (Philippi, 1849)

Tectura fascicularis (Menke, 1851)

Tectura filosa (Carpenter, 1865)

Trimusculus stellatus (Sowerby, 1835)

Crucibulum scutellatum (Wood, 1828)

Diodora inequalis (Sowerby, 1835)

Fissurella oscura Sowerby, 1835

Undulostrea megodon (Hanley, 1846)

Siphonaria maura Sowerby, 1835

\begin{tabular}{|c|c|c|}
\hline Densidad org. $/ \mathrm{m}^{2}$ & Dominancia & Dominancia acumulada \\
\hline 128.67 & 55.311 & 55.311 \\
\hline 26.23 & 11.275 & 66.586 \\
\hline 23.17 & 9.960 & 76.546 \\
\hline 14.39 & 6.186 & 82.732 \\
\hline 7.40 & 3.181 & 85.913 \\
\hline 5.76 & 2.476 & 88.389 \\
\hline 5.46 & 2.347 & 90.736 \\
\hline 3.68 & 1.582 & 92.318 \\
\hline 3.24 & 1.393 & 93.711 \\
\hline 3.10 & 1.333 & 95.044 \\
\hline 2.18 & 0.937 & 95.981 \\
\hline 1.86 & 0.800 & 96.780 \\
\hline 1.71 & 0.735 & 97.515 \\
\hline 1.51 & 0.649 & 98.164 \\
\hline 1.10 & 0.473 & 98.637 \\
\hline 0.80 & 0.344 & 98.981 \\
\hline 0.59 & 0.254 & 99.235 \\
\hline 0.47 & 0.202 & 99.437 \\
\hline 0.35 & 0.150 & 99.587 \\
\hline 0.18 & 0.077 & 99.665 \\
\hline 0.14 & 0.060 & 99.725 \\
\hline 0.11 & 0.047 & 99.772 \\
\hline 0.10 & 0.043 & 99.815 \\
\hline 0.08 & 0.034 & 99.850 \\
\hline 0.07 & 0.030 & 99.880 \\
\hline 0.07 & 0.030 & 99.910 \\
\hline 0.05 & 0.021 & 99.931 \\
\hline 0.03 & 0.013 & 99.944 \\
\hline 0.03 & 0.013 & 99.957 \\
\hline 0.03 & 0.013 & 99.970 \\
\hline 0.03 & 0.013 & 99.983 \\
\hline 0.02 & 0.009 & 99.991 \\
\hline 0.01 & 0.004 & 99.996 \\
\hline 0.01 & 0.004 & 100.000 \\
\hline
\end{tabular}


trabajos como los de Hernández y Acevedo (1987), Acevedo et al. (1987), León (1989), Acevedo et al. (1990), Acevedo y Escalante (1993) y Enciso et al. (1998), la mayoría de los $P$. pansa se encontraron con valores mayores a los $20 \mathrm{~mm}$. Los resultados hallados en Playa Ventura indicaron que la población de caracoles de este sitio, se caracterizó por presentar tallas pequeñas y densidades altas.

Michel-Morfín et al. (2002), mencionan que las mayores tallas promedio e individuales, se encuentran hacia el sur del país y que esto pudiera deberse a la afinidad del caracol al ambiente tropical, sin embargo, las observaciones realizadas por Flores (1995) para Acapulco, Guerrero y los resultados hallados en Playa Ventura con respecto a la densidad y la estructura de tallas, contrasta con el análisis de los autores antes mencionados y se puede considerar la posibilidad de que en el Estado de Guerrero, se presenten las condiciones ambientales favorables para la existencia de densidades altas y tallas pequeñas.

En Playa Ventura, los datos de peso de la población del $P$. pansa fueron los que mayor variación presentaron, dado que los valores el coeficiente rebasaron el $100 \%$, las hembras presentaron los mayores cambios, registrando el menor coeficiente de variación en peso en la recolecta de junio. En marzo de 2001, se observó la mayor actividad de cópula en los caracoles, si se relaciona esta observación con las fluctuaciones del coeficiente de variación en peso de las hembras, se advertirá que los valores mínimos del coeficiente de variación se presentaron posterior a la época de mayor actividad sexual, lo que refiere a que estos valores deben de estar relacionados con la disminución en peso debido de la evacuación de los productos del desove. Esto es coincidente con Ehrhardt (1981), que menciona que puede haber cambios en el peso, aumentando este durante la época de desove y pérdida repentina del mismo al tiempo inmediatamente después de la evacuación de los productos del desove.

En Playa Ventura, se observó reclutamiento de P. pansa prácticamente durante todo el año, siendo mayor en las recolectas de diciembre de
2000 y 2001. Lo hallado en este trabajo en cuanto al reclutamiento coincide con lo observado por Michel-Morfín et al. (2000) para Jalisco y Enciso et al. (1998) para Sinaloa. Por otra parte Álvarez (1989), menciona que la aparición de los reclutas en Michoacán fue en agosto, además de que existen reclutamientos extratemporales. Por otra parte, Hernández y Acevedo (1987), reportaron reclutamiento en julio, agosto y septiembre; Acevedo et al. (1990) señalan la existencia de reclutamiento para machos en marzo y junio y para hembras en mayo y más evidentemente en enero y marzo.

En lo que respecta a la riqueza y a la proporción de especies de moluscos por clase, Castillo-Rodríguez y Amezcua-Linares (1992) en bahía se Santa Cruz, Oaxaca, identificaron 30 especies, donde los gasterópodos fueron mayoritarios proporcionalmente, seguidos por los bivalvos y por último los poliplacóforos. Salcedo et al. (1988), en Zihuatanejo, Guerrero, determinaron 37 especies de moluscos mesolitorales y reportaron a los gasterópodos como la clase mejor representada y García (1994) en Isla Roqueta, Acapulco, determinó 36 especies de moluscos y halló una proporción por clases de moluscos, donde los gasterópodos también fueron mayoritarios. La riqueza y la proporción de especies de moluscos por clase encontrada en los muestreos realizados en Playa Ventura, es similar a lo reportado por los autores antes citados.

Álvarez (1989), reportó para Michoacán a Littorina modesta, L. aspera, Chama squamuligera Pilsbry y Lowe, 1932, Siphonaria palmata Carpenter, 1857, Fissurella nigrocincta Carpenter, 1856, C. articulatus y N. scabricosta, como las especies que principalmente se asocian P. pansa. Castillo-Rodríguez y Amezcua-Linares (1992), encontraron en Oaxaca asociados al caracol púrpura a los géneros Nerita y Littorina, Brachidontes y Choromitylus; Ramos-Cruz (1993) en las bahías de Huatulco, Oaxaca, halló a N. scabricosta, L. modesta, L aspera, Mytilus spp. y Thais spp. Flores (1995), para Acapulco, Guerrero señaló que la asociación característica del caracol púrpura es con L. modesta, S. palmata, H. hansi y C. articulatus. 
De las especies que mencionan los autores antes citados, $C$. squamuligera, $S$. palmata y $F$. nicrocincta, no se encontraron como parte de las especies dominantes asociadas al $P$. pansa en Playa Ventura.

\section{AGRADECIMIENTOS}

Nuestro especial agradecimiento al Consejo Nacional de Ciencia y Tecnología (CONACYT), México. A Gorgonio Ruiz Campos de la Universidad Autónoma de Baja California, por sus valiosas sugerencias y comentarios. A Antonio Leija Tristán de la Universidad Autónoma de Nuevo León, por su apoyo.

\section{RESUMEN}

Estudiamos la demografía de Plicopurpura pansa en Playa Ventura, Guerrero, México, con base en muestreos trimestrales. Su densidad fue de 5.19 caracoles $/ \mathrm{m}^{2}$. La proporción sexual fue cercana al 1:1. La talla promedio fue de $20.10 \mathrm{~mm}$. Las tallas mejor representadas en hembras fueron de 10.5 a $27.0 \mathrm{~mm}$ y en machos de 12.0 a $27.0 \mathrm{~mm}$. La población es de alta densidad y mayor frecuencia de tallas pequeñas. Hubo diferencias entre las tallas promedio de cada muestreo, formándose dos grupos de medias para cada sexo. El peso promedio fue $1.79 \mathrm{~g}$. Las hembras presentaron mayor variación en peso, lo cual podría estar relacionado con el ciclo reproductivo. Hubo reclutamiento en cinco fechas de recolección. El mayor número de cópulas se observaron en marzo de 2001. Se identificaron 26 especies de gasterópodos, siete de bivalvos y una de poliplacóforos. La especie con mayor dominancia fue Brachidontes semilaevis.

Palabras clave: Plicopurpura pansa, población, densidad, tallas, reclutamiento, comunidad malacológica.

\section{REFERENCIAS}

Acevedo, J., E. Hernández. \& D.V. Turcott. 1987. Informe sobre la factibilidad de explotación del tinte de Purpura pansa GOULD, 1853 en la costa de Michoacán. Dirección General de Culturas Populares, Departamento de Programas Científicos y Tecnológicos, México DF. 17 p.
Acevedo, J., M. A. Escalante \& C. López. 1990. Aspectos poblacionales del caracol de tinte Purpura pansa (Gould, 1853), en las costas de Nayarit. Universidad Autónoma de Sinaloa, Rev. Inter. 1: 18-22.

Acevedo, J. \& M. A. Escalante. 1993. Análisis de la población de Purpura pansa en la costa de Sinaloa. Universidad Autónoma de Nuevo León, Facultad de Ciencias Biológicas, Sociedad Mexicana de Zoología. XII del Congreso Nacional de Zoología, Resúmenes. San Nicolás de los Garza, Nuevo León, México: 31.

Acevedo, J., M. A. Escalante \& M. Turok. 1996. El caracol Púrpura. p. 1071-1100. In Pesquerías Relevantes de México. XXX Aniversario del Instituto Nacional de la Pesca. 1962-1992. Secretaría del Medio Ambiente, Recursos Naturales y Pesca. México DF.

Álvarez, D. A. 1989. Relaciones ecológicas y algunos aspectos poblacionales del caracol Purpura pansa Gould, 1853 en la costa del Estado de Michoacán, México. Tesis de Licenciatura, Universidad Michoacana de San Nicolás de Hidalgo, Michoacán México. 123 p.

Anónimo. 1988. Acuerdo Intersecretarial. Diario Oficial de la Federación, Órgano del Gobierno Constitucional de los Estados Unidos Mexicanos. México DF. 10 p.

Anónimo. 1994. Acuerdo Intersecretarial. Diario Oficial de la Federación, Órgano del Gobierno Constitucional de los Estados Unidos Mexicanos. Norma Oficial Mexicana NOM-059-ECOL-1994, 16 de mayo. México DF. 12 p.

Castillo-Rodríguez. Z. G. \& F. Amezcua-Linares. 1992. Biología y aprovechamiento del caracol morado Plicopurpura pansa (Gould 1853) (Gastropoda: Neogastropoda) en la costa de Oaxaca, México. An. Inst. Cienc. Del Mar y Limnol. 19: 223-234.

Daniel, W.W. 2002. Bioestadística. Base para el análisis de las ciencias de la salud. Limusa-Wiley. México DF. 755 p.

Ehrhardt, N. M. 1981. Curso sobre métodos de evaluación de recursos y dinámica de poblaciones. Parámetros poblacionales. FAO-CICIMAR. México DF. 134 p.

Enciso, E. C., V. M. Ramírez, A. R. Tirado \& A. Vallarta. 1998. Evaluación de la población y épocas de reproducción del caracol Purpura pansa (Gould, 1853) en Mazatlán, Sinaloa, México. Tesis de Licenciatura, Universidad Autónoma de Sinaloa. Mazatlán, Sinaloa, México. 56 p. 
Flores, R. P. 1995. Evaluación Biológico-Pesquera del caracol de tinte Purpura pansa (Gould, 1853) del litoral rocoso del municipio de Acapulco, Guerrero, México. Universidad Autónoma de Guerrero, Acapulco, Guerrero, México. 47 p.

García, L. J. 1994. Fauna malacológica de acompañamiento del caracol Purpura pansa Gould, 1853 en la zona mesolitoral de la Isla Roqueta, Acapulco, Guerrero, México. Tesis de Licenciatura, Universidad Autónoma de Guerrero. Acapulco Guerrero, México. 97 p.

Hernández, C. E. \& J. Acevedo. 1987 Aspectos Poblacionales y Etnobiológicos del Caracol Purpura pansa, Gould, 1853 en la Costa de Oaxaca. Tesis de Licenciatura. Facultad de Ciencias. Universidad Nacional Autónoma de México. México DF. 147 p.

Holguín, Q. O. 1993. Distribución, abundancia y composición peso-talla de Purpura pansa (MolluscaGastropoda) en Isla Socorro, Archipiélago Revillagigedo, México. Escuela Nacional de Ciencias Biológicas. Instituto Politécnico Nacional. Zool. Informa. 25: 24-33.

Keen, A. M. 1971. Sea Shells of Tropical West America. Stanford University, Stanford, California, EEUU. 1064 p.

Krebs, C. J. 1999. Ecological Methodology. Addison Wesley Longman, Menlo Park, California, EEUU. $620 \mathrm{p}$.

León, A. H. 1989. Estructura poblacional, producción y tiempo de recuperación del tinte de Purpura pansa Gould, 1853 (Gastropoda: Thaididae) en algunas playas rocosas de la Bahía de Cuastecomate, San Patricio Melaque, Jalisco, México. Tesis de Licenciatura. Facultad de Ciencias. Universidad de Guadalajara. Guadalajara, Jalisco, México. 107 p.

Michel, M. J. E. 2000. Ecología y aprovechamiento del caracol de tinte Plicopurpura pansa en las costas del Pacífico Mexicano. Tesis Doctoral, Instituto Politécnico Nacional. La Paz, Baja California Sur, México. 129 p.

Michel-Morfín, J. E., A. Chavez \& V. Landa. 2000. Population parameters and dye yield of the purple snail Plicopurpura pansa (Gould, 1853) of west central Mexico. J. Shellfish Res. 19: 919-925.
Michel-Morfín, J. E., A. Chavez,. \& L. González. 2002. Estructura de la población, esfuerzo y rendimiento del tinte del caracol Plicopurpura pansa (Gould, 1853) en el Pacífico Mexicano. Cien. Marinas. 28: 357-368.

Morris, P. 1966. Pacific Coast Shells. Peterson Field Guide, Boston, Massachussetts, EEUU. 297 p.

Ramírez-Rodríguez, M. \& L. Naegel. 2003. Crecimiento del caracol de tinte Plicopurpura pansa en Baja California Sur, México. Cien. Marinas. 29: 283-290.

Ramos, C. S. 1993. Abundancia y estructura poblacional del P. pansa (Gould, 1853) en el área de Huatulco, Oaxaca, México (nota científica). Centro Regional de Investigación Pesquera, Salina Cruz, Oaxaca. Secretaría de Pesca. Instituto Nacional de la Pesca. México. 7 p.

Reyes, A. S. C. 1993 Estimación poblacional, producción, foto-oxidación y rendimiento del tinte del caracol Purpura pansa (Gould, 1853) de la zona sur del litoral rocoso de Jalisco. Tesis de Licenciatura. Facultad de Ciencias. Universidad de Guadalajara, Guadalajara, Jalisco, México. 115 p.

Reyes-Gómez, A. \& M. A. Salcedo-Vargas. 2002. The Recent Mexican Chiton (Mollusca:Polyplacophora) Species. Festivus 34: 17-27.

Salcedo-Martínez, S., G. Green, A. Gamboa-Contreras \& P. Gómez. 1988. Inventario de macroalgas y macroinvertebrados bénticos, presentes en áreas rocosas de la región de Zihuatanejo, Guerrero, México. An. Inst. Cienc. del Mar Limnol. 15: 73-96.

Scheaffer, R. L., W. Mendenhall \& L. Ott. 1987. Elementos de muestreo. Iberoamericana, México DF. 321 p.

Skoglund, K. 1991. Additions to the Panamic Province Bibalve (Mollusca) Literature 1971 to 1990 . Festivus 22 (Suppl. 2): 63.

Skoglund, K. 1992. Additions to the Panamic Province Gastropods (Mollusca) Literature 1971 to 1992. Festivus 24 (Suppl. 1): 169.

Steel, R. G. D. \& J. H. Torrie. 1997. Bioestadística: Principios y Procedimientos. Mc. Graw-Hill/ Interamericana de México, México DF. 622 p. 
Stephenson, T. A. \& A. Stephenson. 1949. The Universal Features of Zonation Between Tidemarks at Rocky Coasts. J. Ecol. 37: 289-305.

Turok, M., A.M. Sigler, E. Hernández, J. Acevedo, R. Lara \& V. Turcott. 1988. El caracol púrpura: Una tradición milenaria en Oaxaca. Dirección General de Culturas Populares, SEP. México DF. 166 p.

Villalpando, C. E. 1986. Diversidad y Zonación de moluscos de superficie rocosa. Isla Roqueta, Acapulco Guerrero.
Tesis de Licenciatura. Facultad de Ciencias. Universidad Nacional Autónoma de México, México DF. 150 p.

\section{REFERENCIA DE INTERNET}

Turok, M. \& J. Acevedo. 1999 Incentive measures for conservation of biodiversity and sustainability: case study of the indigenous traditions in México. UNEP. http://www.biodiv.org/doc/case-studies/cs-inc-mxmixteca-en.pdf. Consultada 03 de febrero de 2004. 